\title{
Bird's-Eye View Provided in Real-time via Drone Improves Spatial Perception for Team Sports
}

Satoshi Miura ( $\square$ miura.s.aj@m.titech.ac.jp )

Tokyo Institute of Technology

Kento Nakagawa

Waseda University

Kazumasa Hirooka

Waseda University

Yuya Matsumoto

Waseda University

Yumi Umesawa

Waseda University

Hiroshi Fujimoto

Waseda University

Kazuyuki Kanosue

Waseda University

Masakatsu Fujie

Waseda University

\section{Research Article}

Keywords: Sports equipment, Ergonomics, Human-machine interface

Posted Date: January 19th, 2021

DOI: https://doi.org/10.21203/rs.3.rs-148092/v1

License: (c) (i) This work is licensed under a Creative Commons Attribution 4.0 International License.

Read Full License 


\title{
Bird's-eye view provided in real-time via drone improves spatial perception for team sports
}

\author{
Satoshi Miura ${ }^{1^{*}}$, Kento Nakagawa ${ }^{2}$, Kazumasa Hirooka ${ }^{3}$, Yuya Matsumoto ${ }^{3}$, Yumi Umesawa ${ }^{4}$, Hiroshi \\ Fujimoto $^{4}$, Kazuyuki Kanosue ${ }^{2}$, Masakatsu G. Fujie ${ }^{3}$
}

${ }^{1}$ Department of Mechanical Engineering, the Tokyo Institute of Technology, 2-12-1, Ookayama, Meguro-ku, 152-8550, Tokyo, Japan
${ }^{2}$ Faculty of Sports Sciences, Waseda University, 2-579-15, Mikajima, Tokorozawa-city, 359-1182, Saitama, Japan
${ }^{3}$ Faculty of Science and Engineering, Waseda University, 3-4-1, Okubo, Shinjuku-ku, 169-8555, Tokyo, Japan
${ }^{4}$ Faculty of Human Sciences, Waseda University, 2-579-15, Mikajima, Tokorozawa-city, 359-1192, Saitama, Japan
*Corresponding author: Satoshi Miura (e-mail: miura.s.aj@m.titech.ac.jp).

ABSTRACT

Sports-assisting technologies have been developed; however, most are to improve performances in individual sports such as ski, batting, and swimming. Few studies focused on team sports which require not only motor ability of individual players but also perceptual abilities to grasp positions of their own and others. In the present study, we aim to validate the feasibility of a visual feedback system for the improvement of space perception in relation to other persons that is necessary. Herein, the visual feedback system is composed of a flying drone that transmits the image to the participant's smart glasses. With and without the system, the participant was able to see his/her own relative position in real time though the glass. Nine participants tried to position themselves on the line between two experimenters $30 \mathrm{~m}$ away from each other, which simulated the situation of a baseball cutoff man. As a result, the error distance between the participants' position and the line significantly decreased when using the system than that without the system. Furthermore, after participants practiced the task with the system the error decreased compared to that before the practice. In conclusion, the real-time feedback system from the bird's-eye view would work for improving the accuracy of space perception.

INDEX TERMS

Sports equipment, Ergonomics, Human-machine interface 
Assistive sports technologies can help users learn how to move their body by providing information about ideal and actual body movements ${ }^{1-4}$. Efficient learning methods may also help users by increasing motivation to engage in sports and other forms of exercise. Therefore, assistive sports technologies can contribute for players to maintain motivating for exercising. In various assistive sports technologies, real-time feedback systems with audio signals have been found to improve learning efficiency ${ }^{56}$. For example, Iwata developed a rhythmic auditory feedback system, in which auditory cues are linked with body motion, to improve baseball bat swing performance ${ }^{7}$. Their system helps players to improve their swing speed and improve their kinetic chain during upper body motion. Visual feedback systems have also been found to be useful for movement learning ${ }^{8-10}$. Rekimoto developed a sports assistant system that uses a drone camera for batting training ${ }^{11}$. The drone hovers behind a participant while they engage in batting and captures a visual image of the participant. Although the flying assistant system is able to give visual feedback regarding batting form to the batter, the impact of this feedback on performance is not yet clear.

So far, assistive sports technologies have mainly focused on improving performance in individual player sports. As far as we have investigated related works, few previous studies have examined the use of such systems in improving the movements of players in team sports. In team sports, all of the players on each team have the same goal, and each player must cooperate with the other players in accurate timing and spatial position. Therefore, it is important for players to quickly understand their position in the playing field relative to others.

In this study, we evaluated the feasibility of a visual feedback system for improving spatial perception in players engaged in team sports (Fig. 1). In this visual feedback system, a camera-equipped drone flew above players as they moved on a baseball field (Fig. 2). The drone transmitted an image of the field to transparent smart glasses worn by players, giving them a bird'seye view of the field in real time. In the experiment, we examined the movement of a baseball player who had the role of 'cutoff man', because the position of the 'cut-off man' in relation to other players is particularly important ${ }^{1213}$. We examined how far players could learn where they were located on the field based on the visual data provided by the drone through the comparison of players' motion among before, during and after the use of the system. The participants walked and stopped on the imaginary line connecting the two experimenters before, during and after using the system (Fig. 3). We validated two points: 1) Whether or not the player's self-localization improves when utilizing the visual feedback system compared to that before utilizing it, and 2) Whether or not effects on the player's self-localization lasted after exposure to the system. Finally, in the discussion, we discussed how effectively bird's eye view data worked for users to understand their self-position within an environment, including referring a cognitive feedback model to attribute our results to known theory.

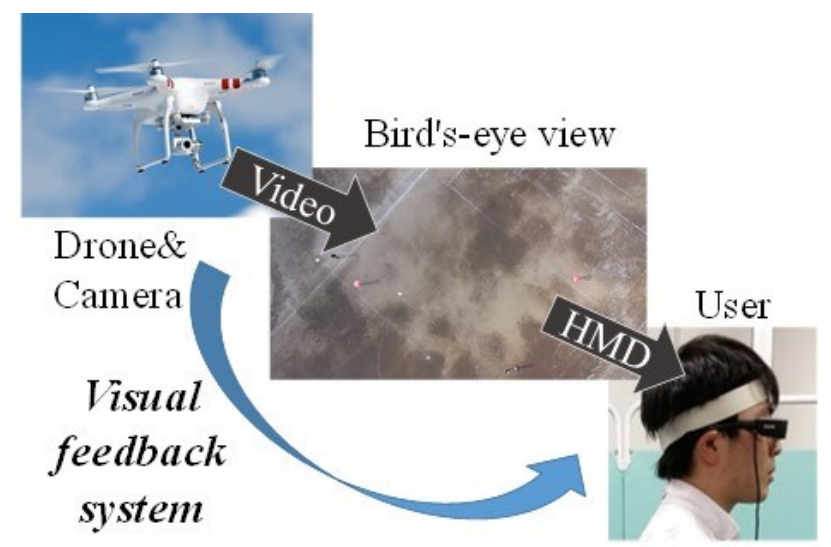

FIGURE 1. Visual feedback system. The drone transmitted the image to the head-mounted display (HMD).

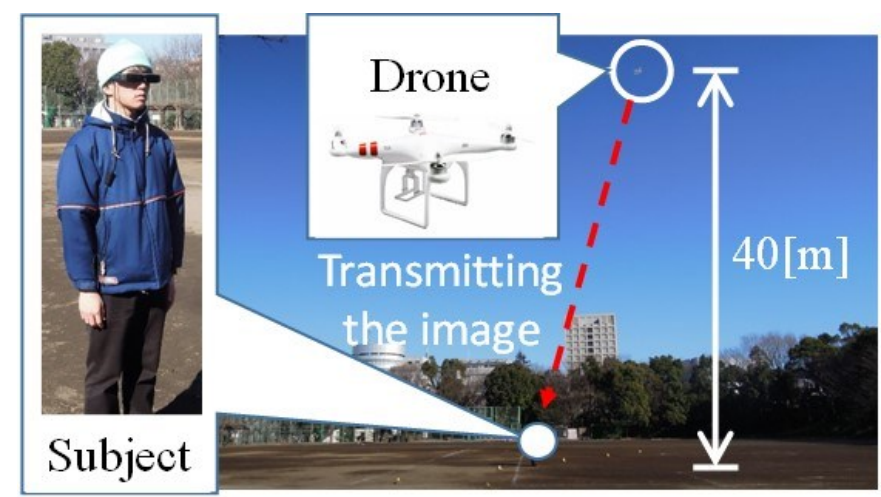

FIGURE 2. Bird's-eye view system. The drone transmitted the camera view to the smart glasses worn by the participant 


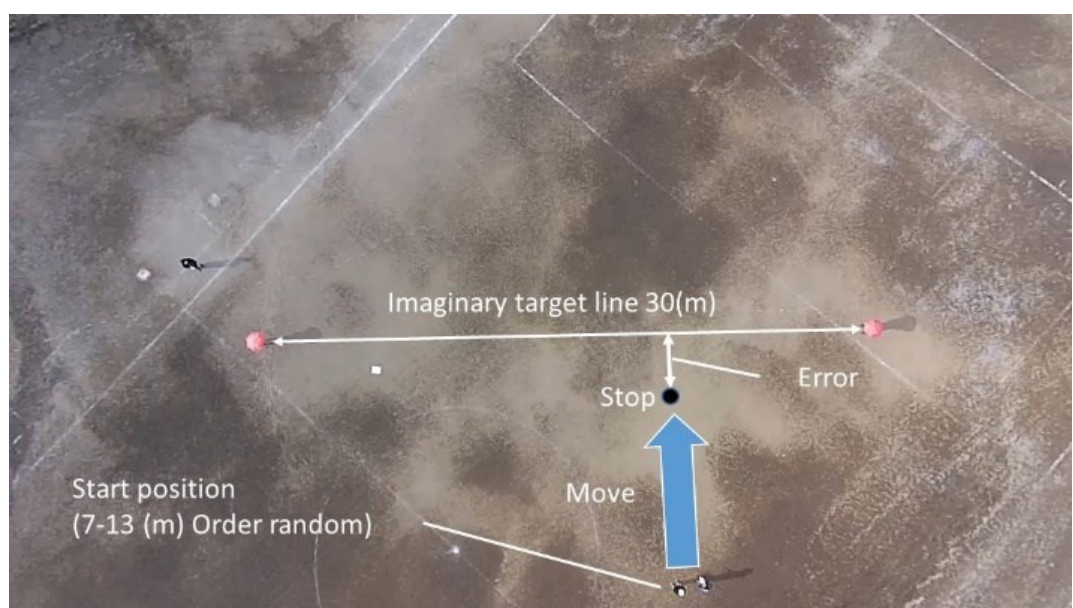

FIGURE 3. The initial position of the participant as seen from the drone camera. The distance between the two experimenters was $30 \mathrm{~m}$. The participants stood at the start point at the beginning of the experiment. The participant was asked to walk quickly with a straight trajectory and then to stop on the imaginary line connecting the two experimenters.

\section{Results}

Fig. 4 shows the relative error with respect to the target line in each participant. We evaluated improvements in spatial perception elicited by the real-time feedback system by measuring the distance error. Fig. 5 shows the (a) absolute and (b) relative error group data. The ANOVA for the absolute error data (Fig. 5 (a)) indicated a significant main effect, as shown in TABLE I $(\mathrm{F}=7.89, \mathrm{p}=0.00232)$. Where F means F-value which is decided by the Degree of freedom in each group on the Fdistribution, and $\mathrm{p}$ means the probability value. We used paired t-tests to compare the error in the trials before, during, and after the trials in which the participant received visual feedback regarding their position $(p<0.05)$, as shown in TABLEs IIIV. The error values in the visual feedback trials were lower than those before or after the feedback trials $(\mathrm{p}<0.01$, effect size $\left.\eta^{2}=0.15, \omega^{2}=0.14\right)$. The ANOVA for relative error data also showed a significant main effect, as shown in TABLE V $(\mathrm{F}=$ $3.54, \mathrm{p}=0.0450)$ and Fig. 5 (b). We used a paired $t$-test to compare the relative error before, during, and after visual feedback $(\mathrm{p}<0.05)$, shown in TABLEs VI-VIII.

TABLE I

RESULTS OF ANOVA FOR ABSOLUTE ERROR

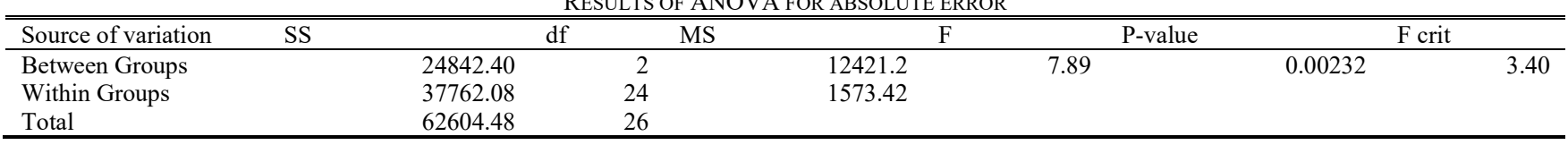

TABLE II

$T$-TEST FOR ABSOLUTE ERROR BEFORE VS. DURING FEEDBACK

\begin{tabular}{|c|c|c|}
\hline & Feedback & \\
\hline Mean [m] & 0.133 & 0.871 \\
\hline Variance $\left[\mathrm{m}^{2}\right]$ & 0.00773 & 0.341 \\
\hline Observations & 9 & 9 \\
\hline Pearson Correlation & 0.0694 & \\
\hline Hypothesized Mean Difference & 0 & \\
\hline Df & 8 & \\
\hline t Stat & -3.79 & \\
\hline $\mathrm{P}(\mathrm{T}<=\mathrm{t})$ one- tail & 0.00265 & \\
\hline t Critical one-tail & 1.86 & \\
\hline $\mathrm{P}(\mathrm{T}<=\mathrm{t})$ two-tail & 0.00531 & \\
\hline t Critical two-tail & 2.31 & \\
\hline
\end{tabular}

TABLE III

RESULTS OF T-TEST FOR ABSOLUTE ERROR DURING VS. AFTER FEEDBACK

\begin{tabular}{|c|c|c|c|}
\hline & Feedback & & \\
\hline Mean [m] & & 0.133 & 0.576 \\
\hline Variance $\left[\mathrm{m}^{2}\right]$ & & 0.00773 & 0.124 \\
\hline Observations & & 9 & 9 \\
\hline Pearson Correlation & & -0.0672 & \\
\hline
\end{tabular}


TABLE IV

$T$-TEST FOR ABSOLUTE ERROR BEFORE VS. AFTER FEEDBACK

\begin{tabular}{|c|c|c|}
\hline & After & \\
\hline Mean $[\mathrm{m}]$ & 0.576 & 0.871 \\
\hline Variance $\left[\mathrm{m}^{2}\right]$ & 0.124 & 0.341 \\
\hline Observations & 9 & 9 \\
\hline Pearson Correlation & 0.615 & \\
\hline Hypothesized Mean Difference & 0 & \\
\hline $\mathrm{df}$ & 8 & \\
\hline t Stat & -1.92 & \\
\hline $\mathrm{P}(\mathrm{T}<=\mathrm{t})$ one- tail & 0.0454 & \\
\hline t Critical one-tail & 1.86 & \\
\hline $\mathrm{P}(\mathrm{T}<=\mathrm{t})$ two-tail & 0.0909 & \\
\hline t Critical two-tail & 2.31 & \\
\hline
\end{tabular}

TABLE V

RESULTS OF ANOVA FOR RELATIVE ERROR

\begin{tabular}{|c|c|c|c|c|c|c|c|}
\hline Source of variation & SS & & $\mathrm{df}$ & MS & $\mathrm{F}$ & P-value & F crit \\
\hline Between Groups & & 24515.54 & 2 & 12257.8 & 3.54 & 0.045 & 3.40 \\
\hline Within Groups & & 83113.51 & 24 & 3463.0 & & & \\
\hline Total & & 107629.04 & 26 & & & & \\
\hline
\end{tabular}

TABLE VI

$T$-TEST FOR RELATIVE ERROR BEFORE VS. DURING FEEDBACK

\begin{tabular}{lrr}
\hline \hline & Before \\
\hline Mean $[\mathrm{m}]$ & Feedback & -0.0287 \\
Variance $\left[\mathrm{m}^{2}\right]$ & 0.0266 & -0.743 \\
Observations & -0.458 \\
Pearson Correlation & 0 \\
Hypothesized Mean Difference & 8 \\
$\mathrm{df}$ & 2.54 \\
$\mathrm{t}$ Stat & 0.0174 \\
$\mathrm{P}(\mathrm{T}<=\mathrm{t})$ one- tail & 1.86 \\
$\mathrm{t}$ Critical one-tail & 0.0347 \\
$\mathrm{P}(\mathrm{T}<=\mathrm{t})$ two-tail & 2.31 \\
$\mathrm{t}$ Critical two-tail & 9 \\
\hline
\end{tabular}

\section{TABLE VII}

RESULTS OF T-TEST FOR RELATIVE ERROR DURING VS. AFTER FEEDBACK

\begin{tabular}{lrr} 
& \\
& Feedback & After \\
\hline \hline Mean $[\mathrm{m}]$ & & -0.0287 \\
Variance $\left[\mathrm{m}^{2}\right]$ & 0.0266 & 9 \\
Observations & -0.358 \\
Pearson Correlation & 0 \\
Hypothesized Mean Difference & 8 \\
df & 0.799 \\
t Stat & 0.224 \\
P(T<=t) one- tail & 1.86 \\
t Critical one-tail & 0.448 \\
P(T<=t) two-tail & 2.31 \\
t Critical two-tail & 9 \\
\hline
\end{tabular}

TABLE VIII

RESULTS OF T-TEST FOR RELATIVE ERROR BEFORE VS. AFTER FEEDBACK

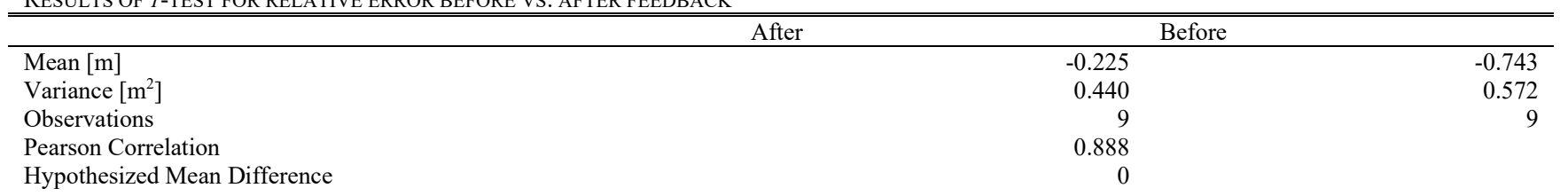




\section{Discussion}

Our results indicate that compared with the trials conducted before the feedback trials, the absolute error in spatial position was lower in the trials with visual feedback and the trials that took place after the visual feedback trials. Furthermore, most participants tended to stop before the line in all conditions (Fig. 5). Paired $t$-tests also showed a significant difference between the stop position in the trials conducted before the feedback condition and zero, which corresponds to perfect performance ( $p$ $<0.05$ ). The results might have been caused by the wide viewing angle in the experiment. As humans usually have a viewing angle of more than $180^{\circ}$, the participant was able to look at the experimenter on each side. However, foveal vision was necessary to determine the correct position. Therefore, determining their own spatial position relative to the line between the two experimenters was not an easy task. In contrast, when they had access to the bird's-eye view from the drone, the participants could look at themselves from an objective viewing angle and confirm his/her own relative position. Therefore, the participant could easily recognize whether they had reached the line in the visual feedback trials.

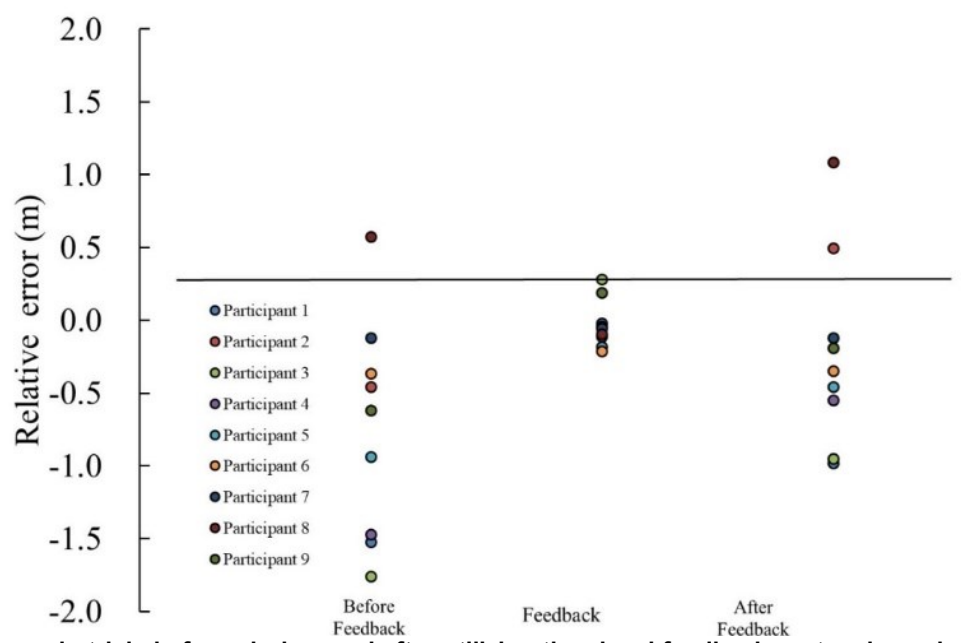

FIGURE 4. Relative error in distance in trials before, during, and after utilizing the visual feedback system in each participant. One plot represents the averaged values of the errors in 18 trials consisting of three trials for each of the six start points. The negative error indicates that the participant stopped on the near side of the line with respect to their starting point. The positive error denotes that they stopped on the far side of the starting point.
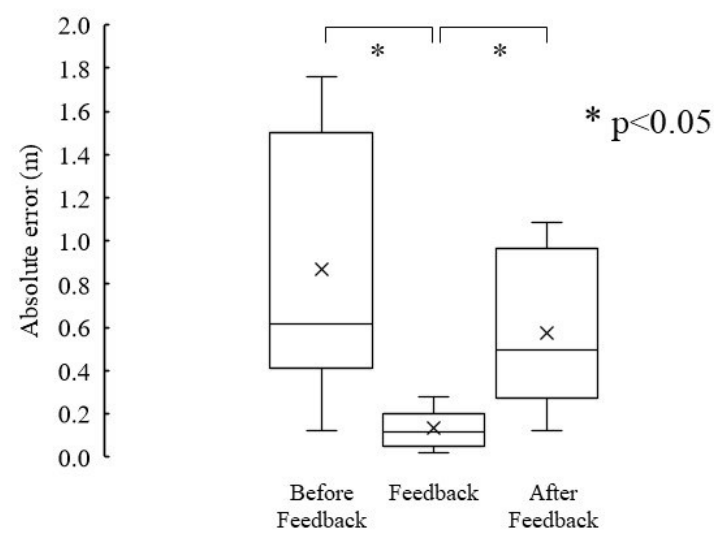

(a) Absolute error 


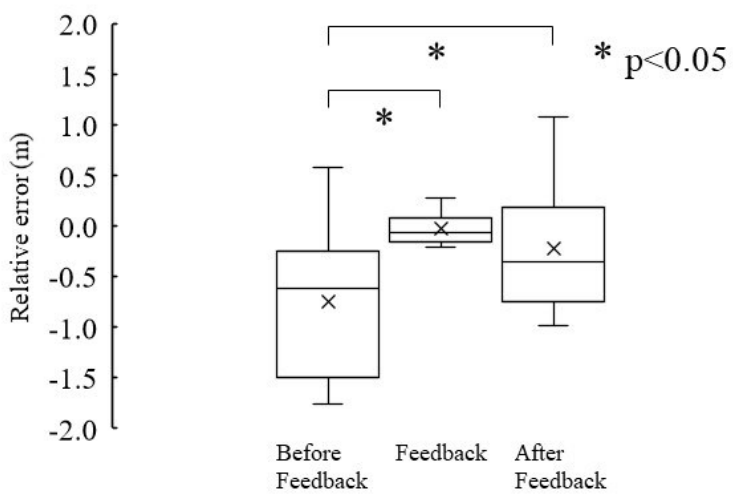

(b) Relative error

FIGURE 5. Averaged error. (a) The absolute error is a measure of the error without a sign. (b) The relative error shows the error with a sign. Negative values indicate that the participant stopped on the near side of the line with respect to their initial position, while positive values indicate that the participant stopped on the far side of the line.

The participants learned from error using the visual feedback system. After the drone system trial, the participants would predict the self-position from the bird's eye view. In the participant's brain, not only the feedback but the feedforward loop would also work for prediction. One of the famous feedforward schemes is the feedback error learning model ${ }^{14}$. Fig. 6 shows the feedback error learning model. The feedback error learning model is the scheme that an inverse model produced based on a feedback loop works as a model for a feedforward loop. After the learning, not only the feedback but feedforward loop can also work. Fig. 6 (a) depicts natural visual feedback without the system. As noted above, this visual feedback is not necessarily accurate. Fig. 6 (b) illustrates the visual feedback given in the smart glasses system. The smart glasses feedback loop was added to the feedback provided by the natural movement of the eyes because the participant was able to see both their natural view and the drone view using the smart glasses. In this system, the participant was able to adjust the inverse model and learn about the error regarding their stopping point. In the trials conducted without the visual feedback system, the participants were not given error feedback and the starting potion was varied. Thus, the practice effect is not likely to explain the observed increase in task accuracy when they had access to the bird's-eye view image.

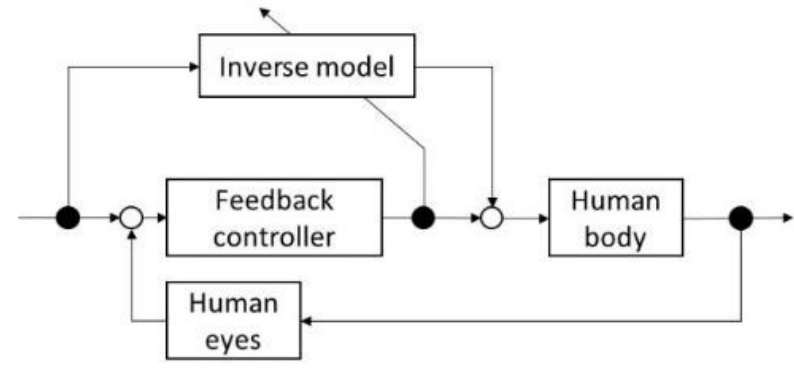

(a) Internal model without the smart glasses system.

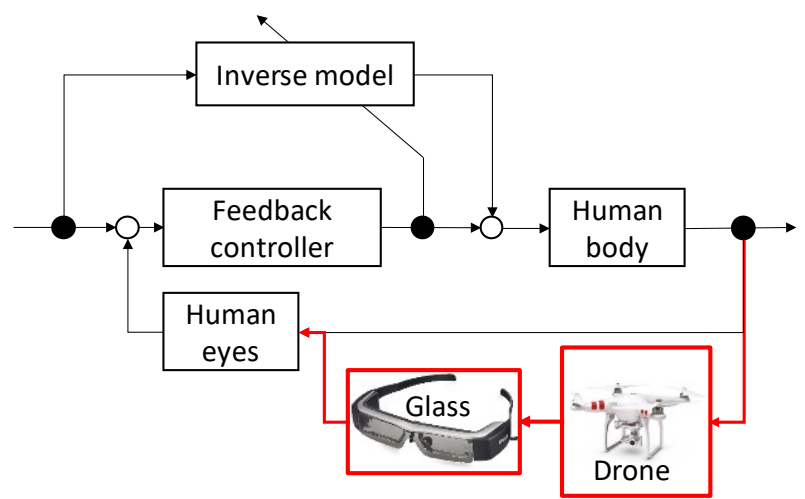

(b) Internal model with the smart glasses system.

FIGURE 6. Feedback error learning model. (a) Visual feedback depends only on the human eyes. (b) Visual feedback depends not only on the human eyes, but also on the system consisting of the drone and the smart glasses. 
The results of this study by themselves are not necessarily sufficient to support the feedback error learning model. For example, in the detail of the inverse model box in Fig. 6, the values in the boxes corresponding to the feedback controller and the human body are unknown because we were not able to quantify the cognitive error. Brain activity measurement would be needed to quantify the amount of cognitive perceptual error. In future work, we hope to study the contents of these boxes via bio-signal analysis.

This study represents a pilot investigation of spatial perception related to sports. In this study, the participant moved but the experimenters did not. In future work, we hope to examine the use of this system for sports in which players must cooperate with other players who are always moving, such as soccer. The real-time visual feedback system could be very useful for situations where both the participants and experimenters are moving simultaneously. In addition, the system could help reduce errors in scoring during real game situations.

Further, AR/VR/mixed reality (MR) could be displayed on the smart glasses. With smart glasses that are transparent, like those used in the present study, the user could simultaneously look at the view from his/her own eyes, a third person or bird'seye view, and an AR/VR/MR display. However, such a display must be designed such that the user can intuitively comprehend complex information. AR/VR/MR applications to sports such as skiing ${ }^{15}$ may enable players to confirm their score in real time while moving. Moreover, this system might enable the creation of new sports. Because the user can access an external viewing angle and their own natural viewpoint, they might be able to move differently. Such new sports might use technology in a way that is similar to e-Sports ${ }^{16}$ or Cybathlon ${ }^{17}$.

\section{Methods}

\section{Participants}

Nine healthy adults (four men and five women with an average age of 21.5 and age range of 20-23 years) participated in the experiment. The participants wore a green cap to enable their position to be easily visible from above, smart glasses, a helmet, and a faceguard for safety. Informed consent was obtained from all participants before the experiment. The experiments were approved by the Waseda University Intuitional Review Board (IRB2014-287). All experiments were performed in accordance with relevant guidelines and regulations. An experimenter who controlled the drone had a Remote Pilot License. The experimenter is called "operator" in this paper.

\section{Bird's-Eye View System}

The image from the drone camera was shown on the smart glasses worn by the participant (Fig. 2). The smart glasses (Moverio, Epson Inc., Tokyo, Japan, weight $88 \mathrm{~g}, 0.42$ wide panel, view angle $23.0^{\circ}$ ) were transparent ${ }^{18}$ so that the participants were able to see the environment through the glasses and the image from the drone camera simultaneously. Thus, they could judge their position from both first-person view and birds-eye-view information (Fig. 7). The image resolution was $960 \times 540$ pixels.

We used the Phantom 2 Vision+ drone manufactured by DJI, Inc. (China). The drone equipped a GNSS receiver and a pneumatic sensor so that it can position itself with 3 degree of freedom in global coordinates. Also, a dedicated console was prepared for users to control it as they like. In this experiment, the drone was controlled to be positioned above participants at a height of $40 \mathrm{~m}$ from the ground. In addition to those, the drone equipped a 14.0-megapixel camera (DJI, 2018) and its realtime images can be transmitted to a smartphone running Android 4.0 (Galaxy, Samson Inc., Korea).

The image from the drone was transmitted to the Moverio smart glasses via the smartphone using Bluetooth at 15.0 fps. Latency, which was less than $200 \mathrm{~ms}$ when the drone was $40.0 \mathrm{~m}$ above the field and less than $130 \mathrm{~ms}$ when it was on the ground, was sufficiently small that the participants did not perceive a time lag ${ }^{19}$. Also, the field of view of the camera was $85.0^{\circ}$, and is sufficient to capture all the field which would be used for this experiment. 


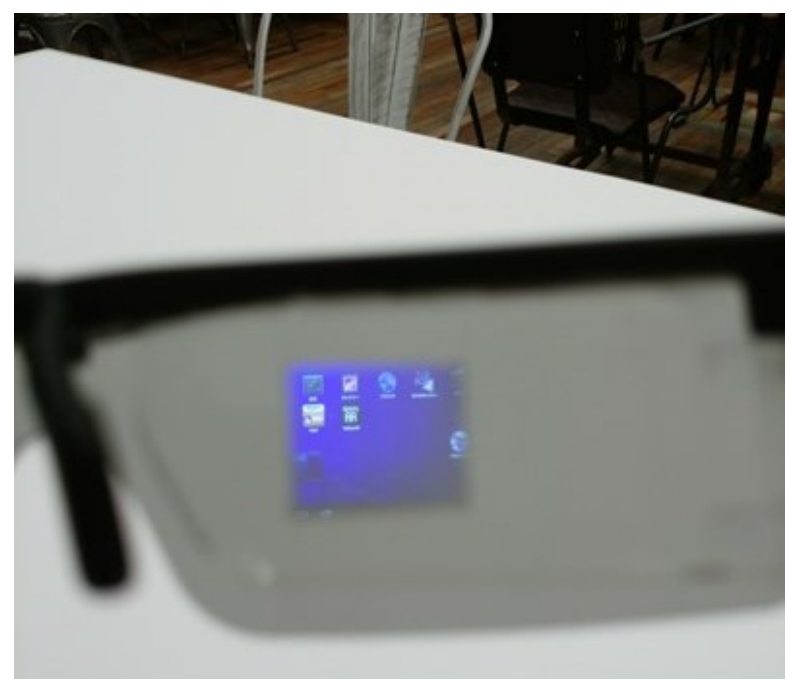

(a) Moverio display

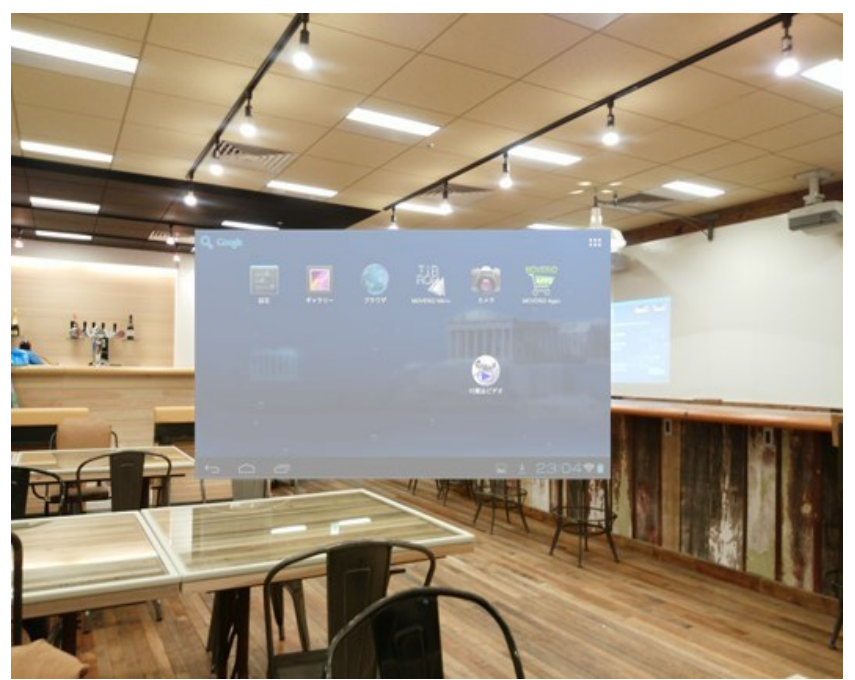

(b) View from Moverio screen

FIGURE 7. Display as seen on the Moverio glasses. It is possible to change the transparency of the display.

\section{Task}

First, participants stood at the start position, and then walked quickly with a straight trajectory towards the imaginary line connecting two experimenters. The two experimenters stood $30 \mathrm{~m}$ apart and held pink umbrellas so that participants could see their position displayed on the smart glasses easily (Fig. 4). The experimenters were standstill during the experiment in order to validate spatial perception ability of the participants relative to the static positions of others. We measured an error as the distance between a participant's stop position and the target line. A third experimenter allocated participants to the initial position, which was randomly varied between 7.0-13.0 m from the target stop line to prevent participants from becoming familiar with the task. Participants were able to look at the positions of the experimenters. To validate feasibility, we selected a short and simple movement task so as to imitate reactions of "cut-off man" s, which require them to locate themselves in only a few seconds in a real game.

\section{Experimental Condition}

We conducted experiments with three conditions in the following order: 1) A participant completed the task without the smart glasses (we call this "before feedback"); 2) the participant wore the smart glasses and completed the task with the birds-eye view image from the drone camera (we call this "feedback"); and 3) as in the first condition, the participant completed the task without the smart glasses (we call this "after feedback"). We evaluated the effect of learning by comparing the results of the "after feedback" trials to those of the "before feedback". There were 6 different start positions that ranged from 7.0-13.0 m with $1 \mathrm{~m}$ pitch from the target line. The 6 start positions were randomized for each of three conditions, with three trials for each start position. Therefore, each participant carried out the task 54 times in total. We did not give feedback to participants regarding error values in each trial.

\section{Analysis}

We used images obtained from the drone camera to measure a participant stop position. Based on colors of a cap and umbrellas, we extracted 1 green point and 2 pink points representing the position of a participant and that of the experimenters respectively from an image. We estimated the distance error according to the number of meters per one pixel using the distance between the two experimenters $(30.0 \mathrm{~m})$ positioned against the long side of the image vertically. The analysis was conducted as follows. 1) Capturing.

The operator adjusted the position of the drone with a display indicator so that the two experimenters with pink umbrellas were captured in the middle region of the camera lens and oriented vertically.

2) Image correction.

The lens distortion in the drone camera image was corrected using Adobe Premier Pro and the DJI Phantom Vision 2+ Preset tool using the field of view $\left(85.0^{\circ}\right)$. Camera image needs to be pre-processed because of distortion on camera lens. The images taken by the drone camera was corrected under the assumption that the distortion can be considered as barrel distortion, which is typical distortion on low magnification camera lens.

\section{3) Extraction.}

Images of the trials were extracted from the video after each experimental session. 


\section{4) Calculation of pixel per meter.}

We recorded the number of pixels between the two umbrellas using Adobe Illustrator. This number of pixels corresponds to $30 \mathrm{~m}$ in the real world. We calculated pixels per meter as a reference based on the number of pixels between the two experimenters.

5) Measurement on pixel.

We then drew a perpendicular line from the participant to the line between the two experimenters. We recorded the number of pixels that made up this perpendicular line.

6) Measurement on meter.

We calculated the distance corresponding to the perpendicular line using the pixels per meter.

We then calculated the error in terms of the distance between the line connecting the two experimenters and the actual stop position of the participant. The error was a negative value when the participant stopped on the near side of the line, and a positive value when the participant stopped on the far side from their initial position. We calculated the relative errors with a sign and the absolute error without a sign. We performed a repeated measure one-way analysis of variance (ANOVA) and a paired $t$-test for the statistical analysis for both error indices.

\section{References}

1. Cui, H. \& Chang, C. Deep Learning Based Advanced Spatiooral Extraction Model in Medical Sports Rehabilitation for Motion Analysis and Data Processing. IEEE Access 8, 115848-115856 (2020).

2. Hsu, Y. L., Chang, H. C. \& Chiu, Y. J. Wearable Sport Activity Classification Based on Deep Convolutional Neural Network. IEEE Access 7, 170199-170212 (2019).

3. Tang, D. Hybridized Hierarchical Deep Convolutional Neural Network for Sports Rehabilitation Exercises. IEEE Access 8, 118969-118977 (2020).

4. Ma, H. \& Pang, X. Research and analysis of sport medical data processing algorithms based on deep learning and internet of things. IEEE Access 7, 118839-118849 (2019).

5. Ghasemzadeh, H. \& Jafari, R. Coordination analysis of human movements with body sensor networks: A signal processing model to evaluate baseball swings. IEEE Sens. J. 11, 603-610 (2011).

6. Cesarini, D. et al. MEDIATION: An eMbEddeD System for Auditory Feedback of Hand-water InterAcTION while Swimming. Procedia Eng. 147, 324-329 (2016).

7. Onishi, T., Yasuda, K., Kawata, S. \& Iwata, H. Development of a rhythmic auditory biofeedback system to assist improving the kinetic chain for bat swing performance. ROBOMECH J. 5, 0-6 (2018).

8. Kim, H. J. \& Kramer, J. F. Effectiveness of visual feedback during isokinetic exercise. J. Orthop. Sports Phys. Ther. 26, 318-323 (1997).

9. Crowell, H. P., Milnert, C. E., Hamill, J. \& Davis, I. S. Reducing impact loading during running with the use of realtime visual feedback. J. Orthop. Sports Phys. Ther. 40, 206-213 (2010).

10. Rice, I., Gagnon, D., Gallagher, J. \& Boninger, M. Hand rim wheelchair propulsion training using biomechanical real-time visual feedback based on motor learning theory principles. J. Spinal Cord Med. 33, 33-42 (2010).

11. Higuchi, K., Shimada, T. \& Rekimoto, J. Flying sports assistant: external visual imagery representation for sports training. in the 2nd Augmented Human International Conference 1-4 (2011). doi:10.1145/1959826.1959833

12. Chueh-Wei, C., Yi-Po, W. \& Hua-Wei, L. An Animation Assisted Training System for the Baseball Cover, Relay and Cutoff Play. Int. J. Comput. Sci. Sport 11, 41-51 (2012).

13. French, K. E., Spurgeon, J. H. \& Nevett, M. E. Expert-Novice Differences in Cognitive and Skill Execution Components of Youth Baseball Performance. Res. Q. Exerc. Sport 66, 194-201 (1995).

14. Katayama, M. \& Kawato, M. Virtual trajectory and stiffness ellipse during multijoint arm movement predicted by neural inverse models. Biol. Cybern. 69, 353-62 (1993).

15. Nakagawa, K. et al. Preliminary Investigation of Real-time Feedback System from the Third-person Perspective Utilizing Augmented Reality AR on Skiing. J. Ski Sci. 15, 77-82 (2018).

16. Martončik, M. E-Sports: Playing just for fun or playing to satisfy life goals? Computers in Human Behavior 48, 208$211(2015)$.

17. Riener, R. The Cybathlon promotes the development of assistive technology for people with physical disabilities. $J$. Neuroeng. Rehabil. 13, 2-5 (2016).

18. Epson. Moverio. (2018).

19. Wang, E. User's Delay Perception and Tolerance in Human-Computer Interaction. Proc. Hum. Factors Ergon. Soc. Annu. Meet. 46, 651-655 (2002). 


\section{Acknowledgment}

The authors wish to thank all the participants. This work was supported in part by a grant from the Institutes of Advanced Active Aging (AAA) in Waseda University and JSPS KAKENHI \#JP18K18405 and JP18H04087. The authors of this paper would like to thank Waseda University in Japan for offering funding support for this publication. We thank Sydney Koke, MFA, from Edanz Group (www.edanzediting.com/ac) for editing a draft of this manuscript.

\section{Author contribution}

SM proposed the study. KN, KH, YM, YU carried out the experiment and analyzed the results. HF, KK, MGF guided the study.

\section{Additional information}

Competing interests: The authors declare no competing interests. 
Figures

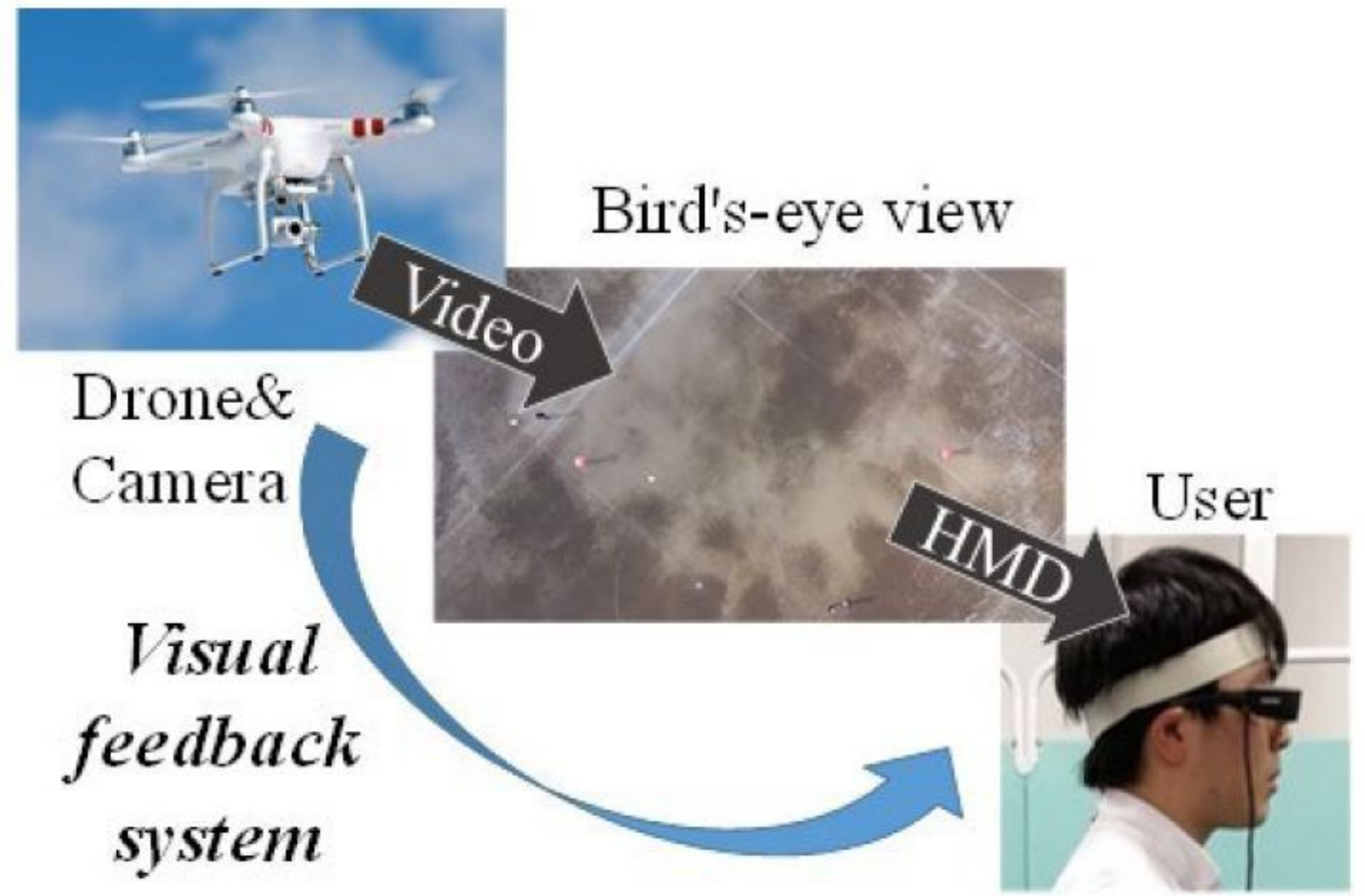

Figure 1

Visual feedback system. The drone transmitted the image to the head-mounted display (HMD). 


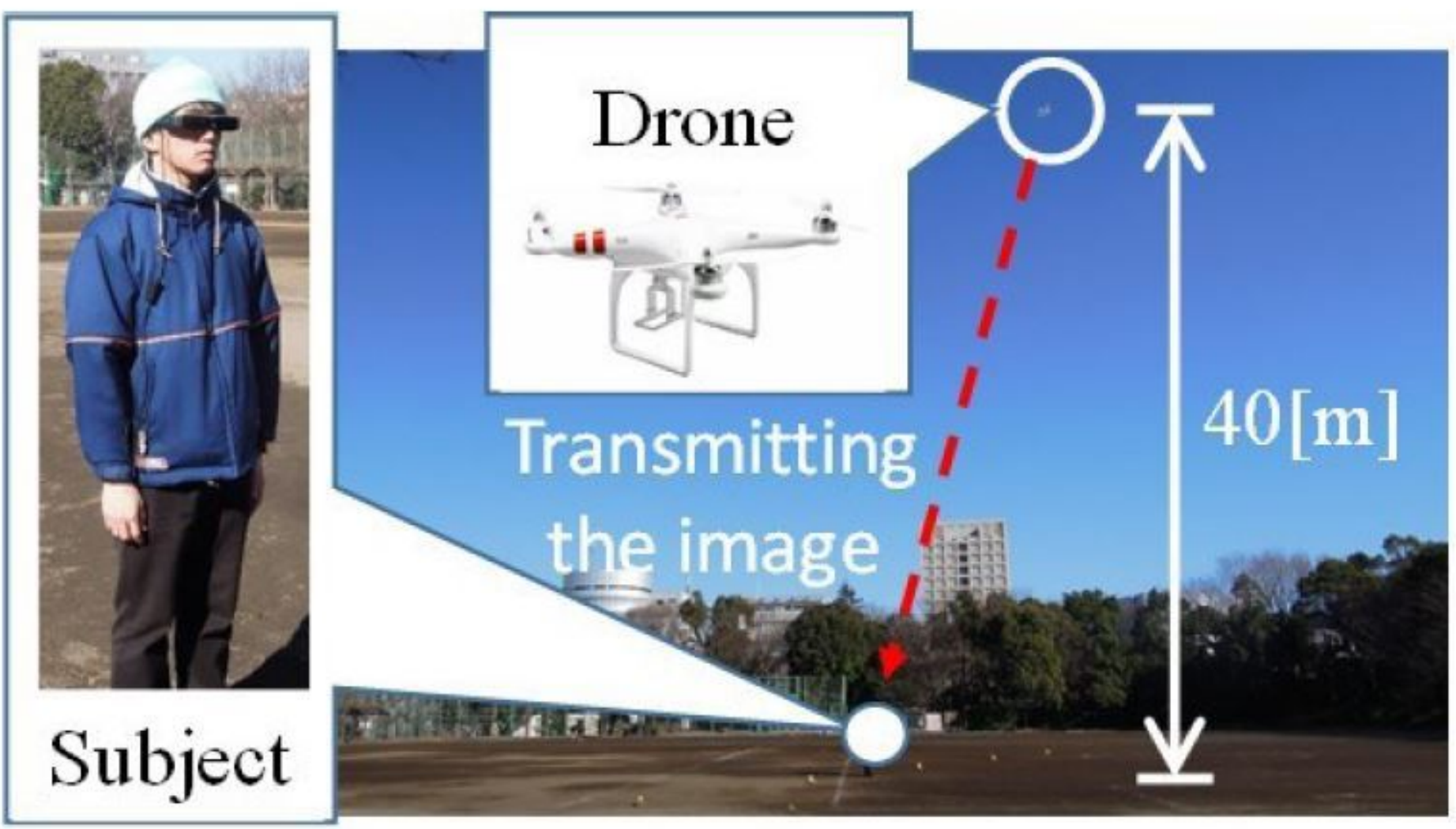

Figure 2

Bird's-eye view system. The drone transmitted the camera view to the smart glasses worn by the participant 


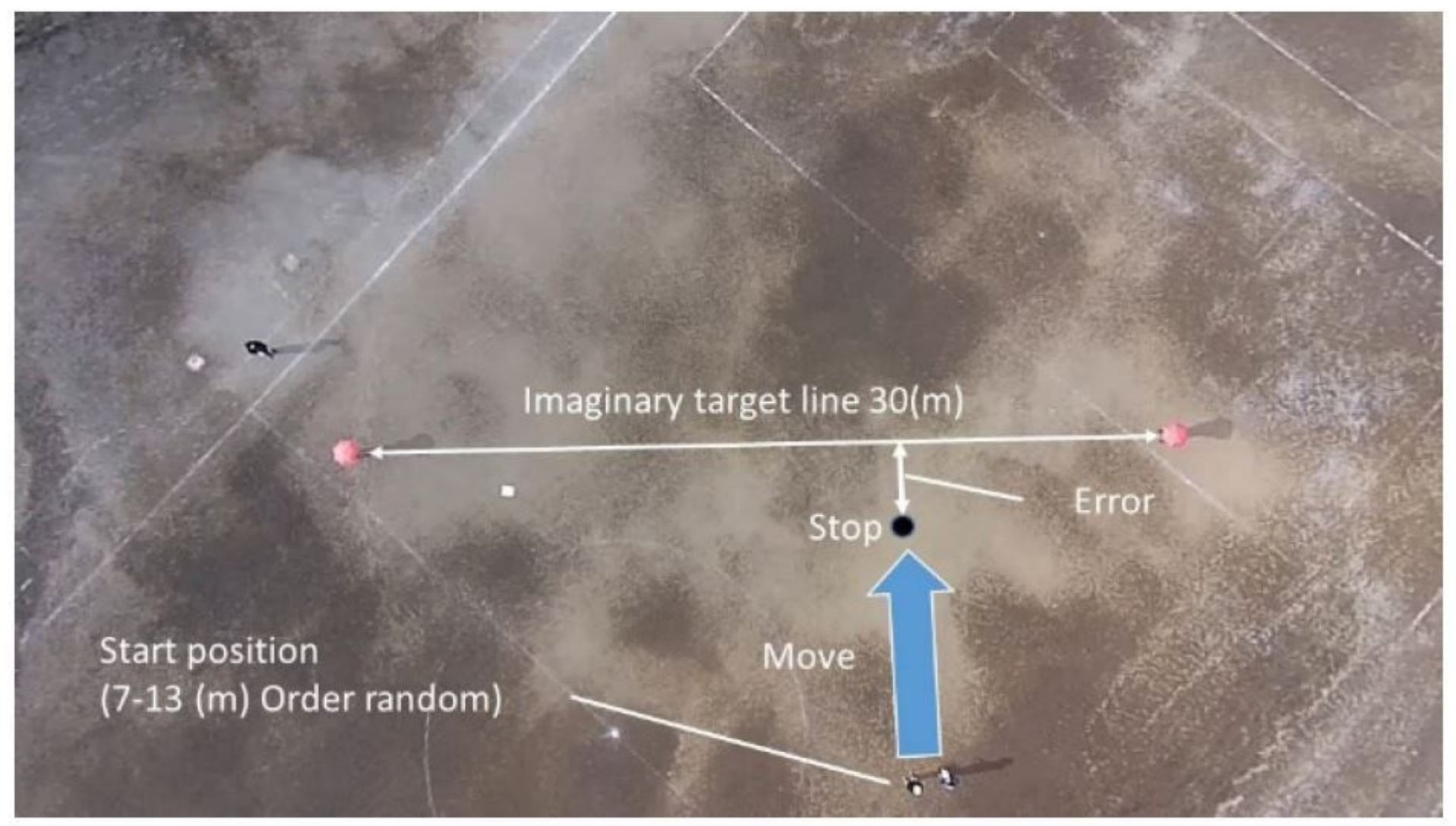

\section{Figure 3}

The initial position of the participant as seen from the drone camera. The distance between the two experimenters was $30 \mathrm{~m}$. The participants stood at the start point at the beginning of the experiment. The participant was asked to walk quickly with a straight trajectory and then to stop on the imaginary line connecting the two experimenters. 


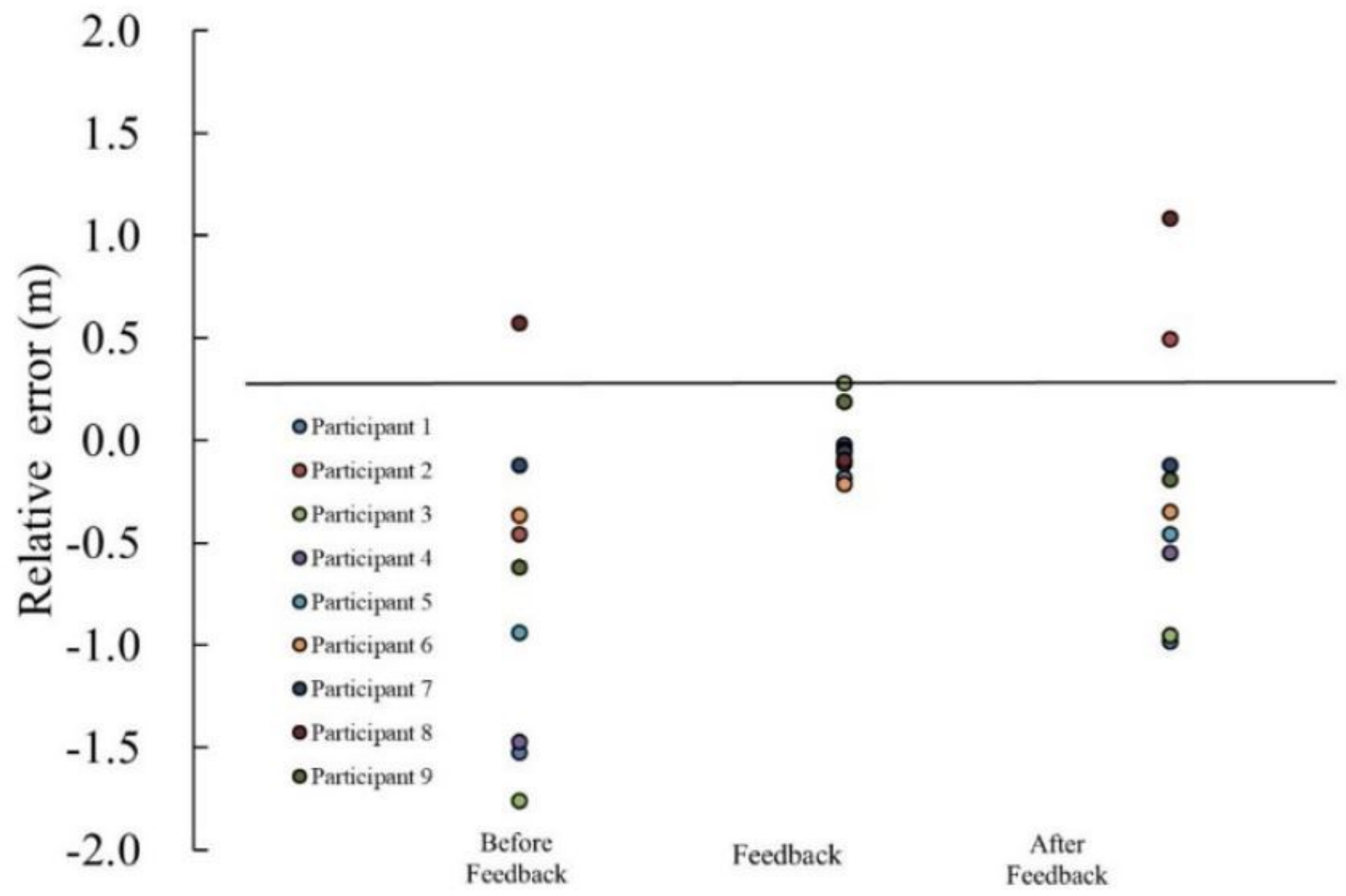

Figure 4

Relative error in distance in trials before, during, and after utilizing the visual feedback system in each participant. One plot represents the averaged values of the errors in 18 trials consisting of three trials for each of the six start points. The negative error indicates that the participant stopped on the near side of the line with respect to their starting point. The positive error denotes that they stopped on the far side of the starting point. 

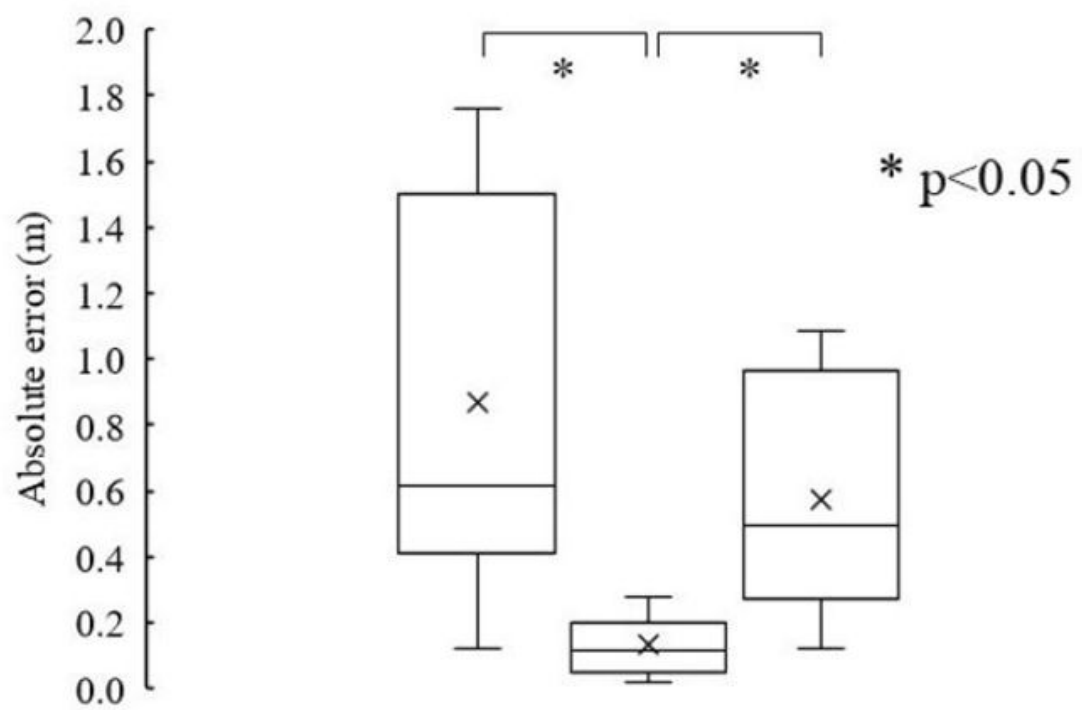

Before
Feedback $\quad$ Feedback $\begin{gathered}\text { After } \\ \text { Feedback }\end{gathered}$

(a) Absolute error

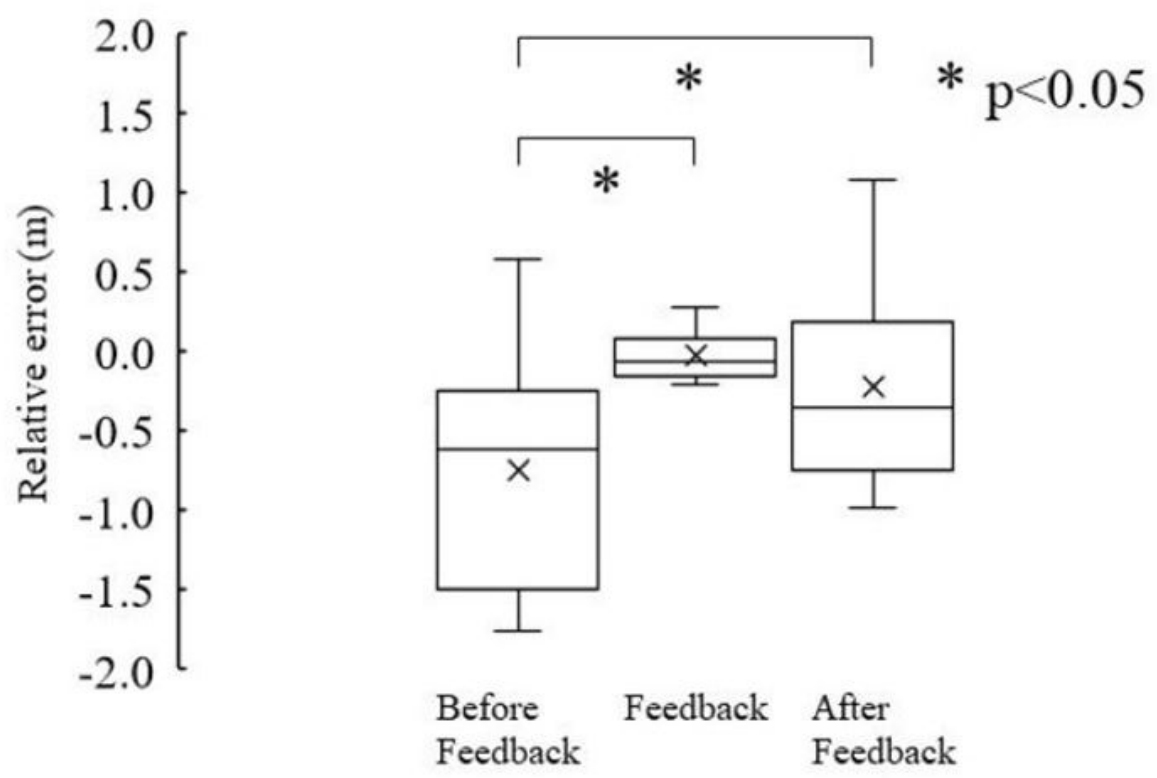

(b) Relative error

\section{Figure 5}

Averaged error. (a) The absolute error is a measure of the error without a sign. (b) The relative error shows the error with a sign. Negative values indicate that the participant stopped on the near side of the line with respect to their initial position, while positive values indicate that the participant stopped on the far side of the line. 


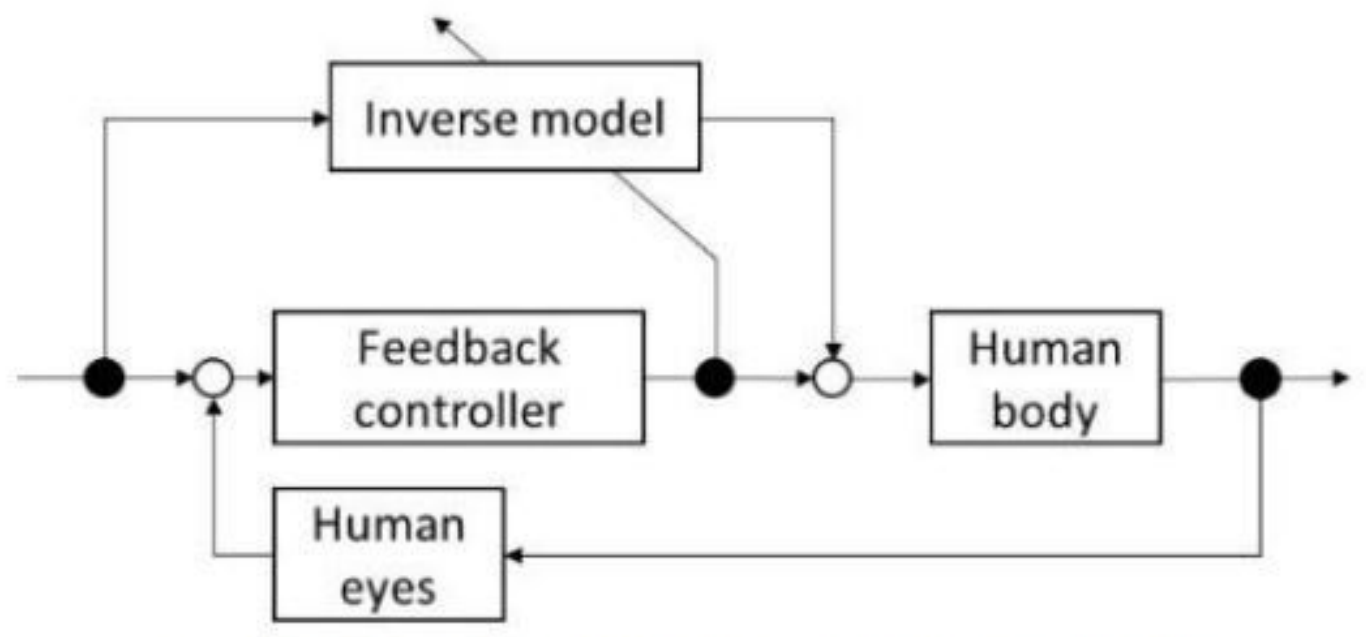

(a) Internal model without the smart glasses system.

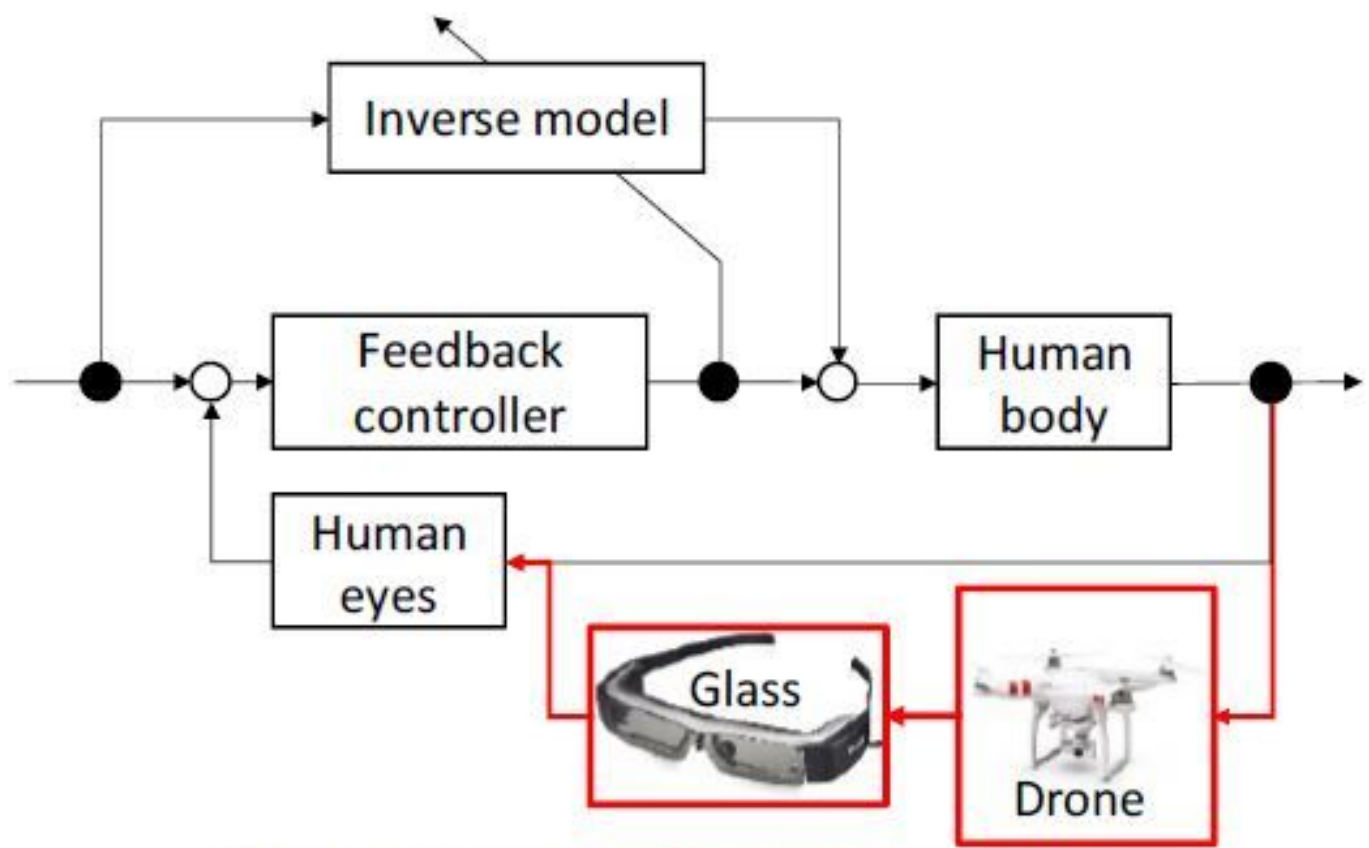

(b) Internal model with the smart glasses system.

Figure 6

Feedback error learning model. (a) Visual feedback depends only on the human eyes. (b) Visual feedback depends not only on the human eyes, but also on the system consisting of the drone and the smart glasses. 


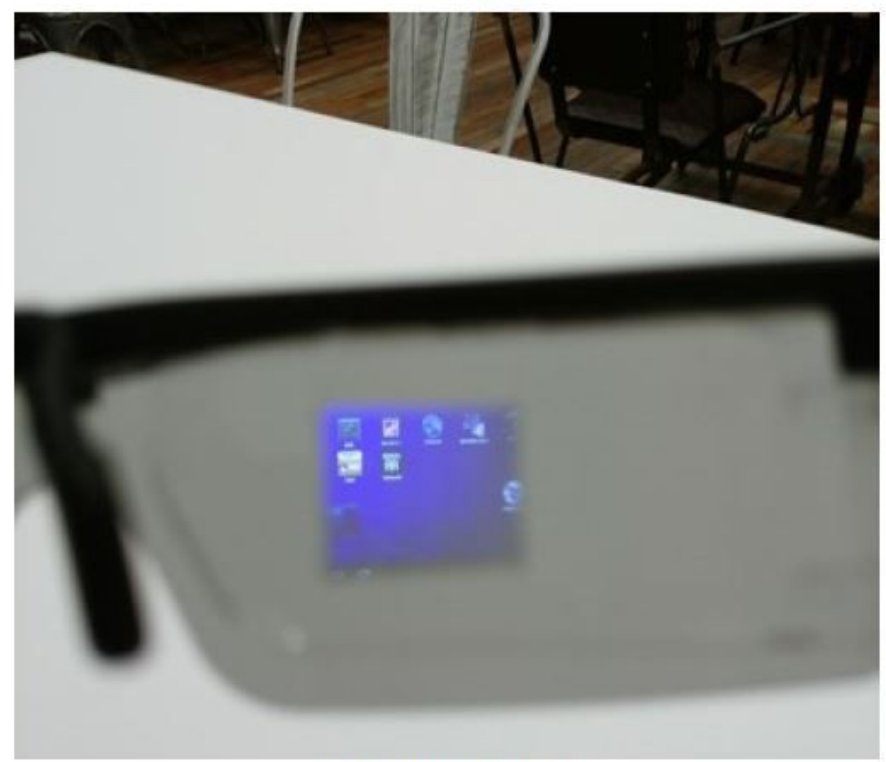

(a) Moverio display

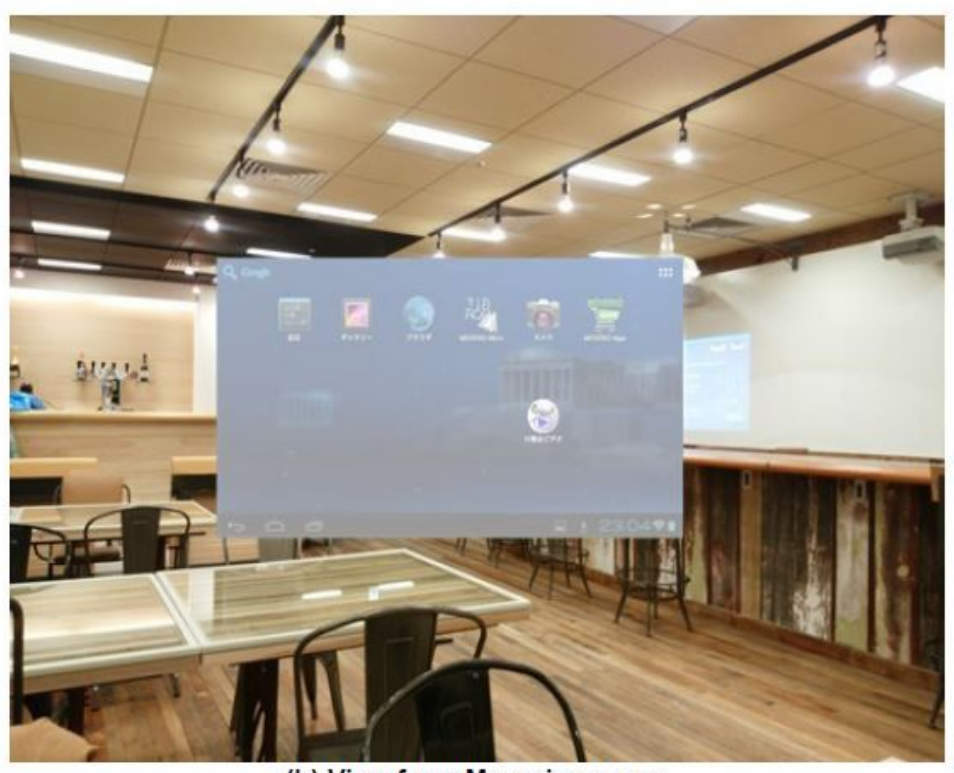

(b) View from Moverio screen

\section{Figure 7}

Display as seen on the Moverio glasses. It is possible to change the transparency of the display. 